\title{
EXTREMAL DISTANCE, HYPERBOLIC DISTANCE, AND CONVEX HULLS OVER DOMAINS WITH SMOOTH BOUNDARY
}

\author{
Matt Feiszli \\ Yale University, Mathematics Department \\ 10 Hillhouse Ave, New Haven, CT 06520-8283, U.S.A.; mattfeiszli@gmail.com
}

\begin{abstract}
Given a simply connected planar domain $\Omega$ we develop estimates for boundary derivatives on $\partial \Omega$ and estimates for hyperbolic and extremal distances in $\Omega$ and the hyperbolic convex hull boundary $S_{\Omega}$. We focus on the case when the underlying domain has smooth boundary; this allows very explicit formulas in terms of a collection of invariants which clarify behavior even in the generic case. In particular, we are able to obtain very explicit estimates using the intimate connection between the convex hull boundary and the geometry of the medial axis. As applications, we include here a refinement and alternate proof of the Thurston-Sullivan conjecture that the nearest-point retraction is 2-Lipschitz in the hyperbolic metrics and a variant of the Ahlfors distortion theorem which works as an integral along branches of the medial axis.
\end{abstract}

\section{Introduction}

In this paper, we study the special case of the hyperbolic convex hull boundary $S_{\Omega}$ over a domain $\Omega \subset \mathbf{C}$ with smooth boundary; in particular we obtain a number of estimates and invariants which relate extremal lengths and hyperbolic distances "upstairs" in the convex hull boundary to the same quantities "downstairs" in the domain via the nearest-point retraction map $r: \Omega \rightarrow S_{\Omega}$. The study of the smooth case was originally motivated, perhaps unexpectedly, by computer vision, where conformal mapping techniques were introduced by Sharon and Mumford (see [25]) to construct metric spaces of smooth curves. In this application, smooth Jordan curves are represented by their associated welding maps, and a Riemannian metric on curves is induced by the Weil-Peterson metric on the diffeomorphism group of the circle. The curves of interest are typically at least $C^{2}$, and careful geometric estimates for boundary derivatives and extremal distances are required in order to understand geodesic distances between curves. We obtain these by working with the convex hull boundary $S_{\Omega}$. The surface $S_{\Omega}$ admits an explicit construction for the Riemann map $\iota: S_{\Omega} \rightarrow \mathbf{D}$ (see, for example, [6] or [18]) and this map $\iota$ can act as a sort of poor-man's Riemann map from the disk to $\Omega$ itself. Among the advantages is that $S_{\Omega}$ is a ruled surface, and this special geometry permits easier analysis; in addition, the Riemann map $\iota$ is an entirely local construction, unlike the global nature of the Riemann map to $\Omega$.

The resulting formulas are, for the most part, simple and explicit, and we feel that working in the smooth case has the potential to simplify many computations

doi:10.5186/aasfm.2011.3612

2010 Mathematics Subject Classification: Primary 30C85; Secondary 31A15, 30C35.

Key words: Harmonic measure, extremal length, convex hulls, hyperbolic 3-manifolds, conformal mappings, medial axis. 
and clarify the geometry even in the generic case; we give a few examples, including a proof and refinement of the Thurston-Sullivan " $L=2$ " conjecture, first proven with sharp constant by Epstein, Marden and Markovic in [17], which states that the nearest-point retraction is hyperbolic 2-Lipschitz. Our result, which we state in a moment, shows the role played by the local bending of the hull, gives an alternate approach to the proof. We also obtain a variant of the Ahlfors distortion theorem, where the famous estimate $\int \frac{d x}{\theta}$ is replaced with an integral along the medial axis of $\Omega$.

We now describe the major results in this paper. Many results rely on the geometry of the medial axis of $\Omega$. The intimate connection between the medial axis and the convex hull boundary was first exploited, to our knowledge, by Bishop (see [7], [6]) in the context of numerical conformal mapping, and our work here owes an obvious debt to his. We also include a short appendix describing the basic results about the medial axis.

The first section of this paper collects some facts and definitions from hyperbolic geometry. In Section 3 we develop explicit estimates that relate hyperbolic distance in $\Omega$ to distances on $S_{\Omega}$; this section includes the following result, relating the hyperbolic distances:

Theorem 1. (Hyperbolic distances comparable) Let $\gamma:[0, T] \rightarrow \Omega$ be path in $\Omega$ and $\sigma=r(\gamma)$. Then the hyperbolic velocities of $\gamma$ and $\sigma$ satisfy

$$
\frac{1}{2}\|\dot{\sigma}\|_{S_{\Omega}} \frac{1+\kappa_{1}}{1+\kappa_{1} \sin \theta} \leq\|\dot{\gamma}\|_{\Omega} \leq\|\dot{\sigma}\|_{S_{\Omega}}\left(1+\kappa_{1} \cos \theta\right)
$$

where $\theta$ is the angle between $\dot{\sigma}$ and the transverse vector to the bending line on which $\sigma$ lies, $\kappa_{1}$ is the hyperbolic principal curvature of $S_{\Omega}$, and the norms $\|\cdot\|_{\Omega}$ and $\|\cdot\|_{S_{\Omega}}$ denote hyperbolic length on $\Omega$ and $S_{\Omega}$.

We also obtain the following result, which shows how the bending influences the local Lipschitz constant of the nearest-point retraction map. $L_{h}$ is hyperbolic distance.

Theorem 2. (" $L=2$ " conjecture) The nearest point retraction map $r: \Omega \rightarrow S_{\Omega}$ is 2-Lipschitz in the hyperbolic metrics. Further, if $\gamma \in \Omega$ is a path whose retract $\sigma=r(\gamma)$ is transverse to any bending lines it encounters, we have

$$
L_{h}[\sigma] \leq 2 L_{h}[\gamma]-\int_{\sigma} d \beta
$$

Our proof differs from that of Epstein, Marden and Markovic in [17] in that they approximate a generic domain with a finite union of disks and we approximate using a domain with real-analytic boundary. In the case of a finite union of disks, the Jacobian is essentially trivial everywhere except on bending lines, where it is singular in the transverse direction. In the real-analytic case, one can talk almost everywhere about the Jacobian of the nearest-point retraction, and one sees that the Jacobian is primarily a function of the bending of the dome $S_{\Omega}$. This makes certain estimates relating bending to various other kinds of distortion, like hyperbolic lengths and extremal distances, very easy to derive.

In Section 4 we obtain the following estimate for extremal distances between pairs of bending lines $B_{s}$ and $B_{t}$ in $S_{\Omega}$ and their preimages $F_{s}=r^{-1}\left(B_{s}\right), F_{t}=r^{-1}\left(B_{t}\right) \subset \Omega$ : 
Theorem 3. (Extremal distance comparable) Let $F_{s}, F_{t} \subset \Omega$ be two foliating arcs. Then

$$
d_{S_{\Omega}}\left(B_{s}, B_{t}\right) \leq d_{\Omega}\left(F_{s}, F_{t}\right) \leq d_{S_{\Omega}}\left(B_{s}, B_{t}\right)+\frac{2}{\pi^{2}} \int_{s}^{t} d \beta,
$$

where $\beta(t)$ is the bending measure associated with $S_{\Omega}$.

This, plus some computation using the medial axis, leads immediately to the following analog of the Ahlfors distortion theorem. If $m$ is the arc-length parameter on a branch of the medial axis (see Appendix) of $\Omega$ with corresponding points of tangency $(a(m), b(m))$, then:

Corollary 4. Let $F_{s}, F_{t}, s<t$ be two foliating arcs in $\Omega$. Then

$$
d_{\Omega}\left(F_{s}, F_{t}\right) \geq \frac{2}{\pi} \int_{s}^{t} \frac{\sqrt{\dot{a} \dot{b}}}{|b-a|} d m .
$$

Acknowledgements. The results are part of my $\mathrm{PhD}$ thesis. I wish to offer my warmest thanks to my advisor David Mumford.

\section{Background: hyperbolic 3-space}

We are interested in conformal structures on a domain $\Omega$ lying on the Riemann sphere $\hat{\mathbf{C}}$. These conformal structures can be approximated using constructions from hyperbolic geometry which we now describe. These hyperbolic constructions have the advantage of being entirely local, unlike the global nature of the Riemann map. We regard $\hat{\mathbf{C}}$ as the boundary at infinity of hyperbolic 3 -space $\mathbf{H}^{3}$. Here we describe the constructions we will need to relate hyperbolic and conformal geometries.

2.1. Models of hyperbolic space. Equipping the open unit ball in $\mathbf{R}^{n}$ with the Poincaré metric $d s^{2}=\left(\frac{2}{1-|z|^{2}}\right)^{2}|d z|^{2}$, where $|z|$ is the distance to the origin and $|d z|$ is the Euclidean distance element, gives a model of hyperbolic space $\mathbf{H}^{n}$. With this metric, the space is normalized so all sectional curvatures are a constant -1 . The isometries of $\mathbf{H}^{n}$ act on the boundary $S^{n-1}$ as conformal automorphisms.

We are particularly interested in the case $n=3$. We regard $\hat{\mathbf{C}}$ as the boundary at infinity of hyperbolic 3-space $\mathbf{H}^{3}$. In this Poincaré model, arcs of circles orthogonal to $\hat{\mathbf{C}}$ are geodesics in the half-space or ball models. The isometries of $\mathbf{H}^{3}$ act on the boundary as the group of linear fractional maps $S L_{2}(\mathbf{C})$. Denote the closed unit ball, with the hyperbolic structure on the interior and conformal structure on the boundary by $\mathbf{B}^{n}$.

One may also realize the same geometry in the half-space model (see Figure 1); the two models are related by a conformal self-map of $\mathbf{R}^{3}$. In this model, hyperbolic 3 -space is the set $\left\{\left(x_{1}, x_{2}, x_{3}\right): x_{3}>0\right\}$ with the metric $d s^{2}=\frac{d x_{1}^{2}+d x_{2}^{2}+d x_{3}^{2}}{x_{3}^{2}}$. Geodesics are again arcs of circles orthogonal to the $\left(x_{1}, x_{2}\right)$ plane $\hat{\mathbf{C}}$, and isometries again extend to the boundary and act conformally on $\hat{\mathbf{C}}$.

\subsection{Convex hulls and the dome.}

Definition 1. (Convex) A non-empty subset $F \subset \mathbf{B}^{n}$ is said to be convex if, given any two points in $F$, the geodesic arc joining them is also contained in $F$. 


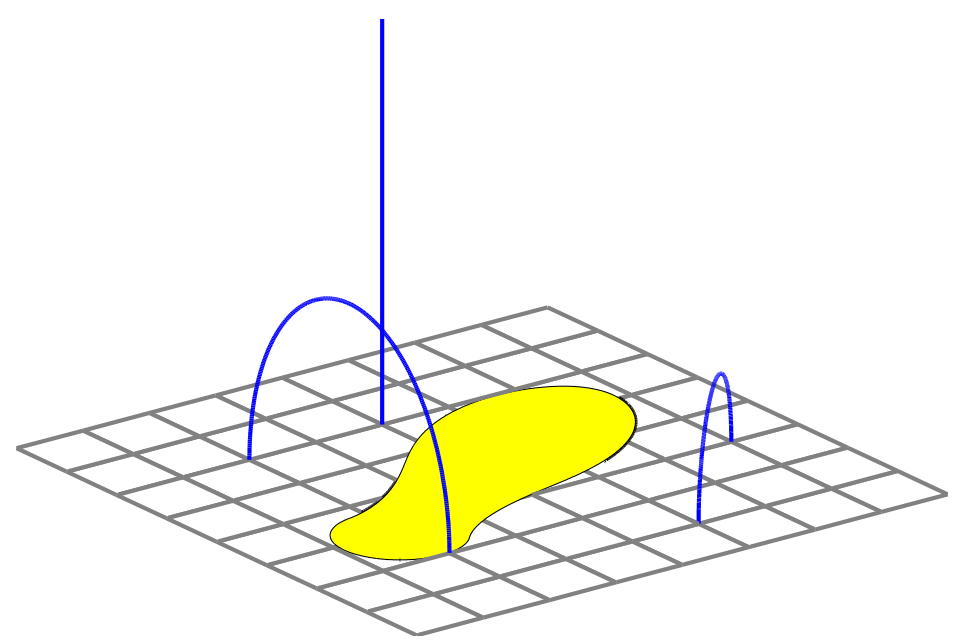

Figure 1. The half-space model of $\mathbf{H}^{3}$ : Arcs of circles orthogonal to $\hat{\mathbf{C}}$ are geodesics. A planar domain lies in $\hat{\mathbf{C}}$, the boundary of $\mathbf{H}^{3}$.

A supporting half-space for a convex set $E \subset \mathbf{B}^{n}$ is a closed half-space containing $E$ whose boundary meets $E$. A support plane for $E$ at some point $z \in E \cap \mathbf{H}^{n}$ is the boundary of a supporting half-space which meets $E$ at $z$. It can be shown (see [12]) that support planes exist for all points in the boundary of $E$.

Given a closed nonempty convex subset $E \subset \mathbf{B}^{n}$, there is a canonical retraction $r: \mathbf{B}^{n} \rightarrow E$. We define it as follows:

Definition 2. (Nearest-point retraction map $r: \mathbf{B}^{n} \rightarrow E$ ) Let $E \subset \mathbf{H}^{n}$ be a convex set. For $z \in \mathbf{H}^{n}$ we define the map $r: H S^{n} \rightarrow \partial E$ as follows:

$$
r(z)=\arg \min _{w \in E} d_{h}(z, w) .
$$

For $z \in \partial \mathbf{H}^{n}$, we define $r(z)$ by inflating a horoball tangent at $z$ until it makes contact with some point $w \in E$ and set $r(z)=w$.

One may also construct the map $r$ by inflating balls centered at $z$ until they make contact with $E$. By convexity of $E$, the ball will have a unique first point of contact in $\partial E$; this unique point is $r(z)$. This geometric construction makes it clear that the extension of $r$ to the boundary of hyperbolic space is natural.

The nearest-point retraction map is both continuous and distance-decreasing; for details and proof, see, e.g., [12].

Definition 3. (Hyperbolic convex hull) The convex hull of a set $F \subset \mathbf{B}^{n}$ is the smallest convex subset of $\mathbf{B}^{n}$ containing $F$.

We will use the notation $C(F)$ for the convex hull of $F \subset \mathbf{B}^{n}$. The notation $\partial C(F)$ refers to the part of the boundary of $C(F)$ which lies in $\mathbf{H}^{n}$.

Definition 4. (The dome of a planar domain) Let $\Omega \subset \partial \mathbf{H}^{3}$ be a proper subset of the plane and $C\left(\Omega^{c}\right)$ be the convex hull of its complement. The "dome" $S_{\Omega}$ is the boundary $\partial C\left(\Omega^{c}\right)$.

We can equip the dome with a natural path metric by restricting the hyperbolic metric in $\mathbf{H}^{3}$ to paths contained in the dome. In fact, the resulting metric is the hyperbolic metric for $S_{\Omega}$. There is also an explicit construction for the Riemann map $\iota: S_{\Omega} \rightarrow \mathbf{D}$, due to Thurston, which we will describe in a moment. 
2.3. Bending. If $\Omega \subset \hat{\mathbf{C}}$ is a simply-connected region which is a union of $N$ disks, the dome $S_{\Omega}$ has a particularly simple form. If we consider all support planes $P$ for $S_{\Omega}$, we see that $P \cap S_{\Omega}$ is either a line or a piece of a hyperbolic plane. If $P \cap S_{\Omega}$ is a line, we call it a bending line; otherwise $P \cap S_{\Omega}$ is called a flat piece. We say that such a dome is finitely-bent. Note that if $z \in S_{\Omega}$ lies in a bending line it has a set of support planes; if $z$ lies in a flat piece it has only one support plane. The bending angle associated with a bending line is angle between the extremal support planes for the line, that is, it is the angle at which the associated flat pieces meet (the angle between their normal vectors).

The bending at each intersection induces a transverse measure on the dome, called the bending measure, constructed as follows. For $z \in S_{\Omega}$, let $\beta(z)$ be the bending angle at $z$. This is zero unless $z$ lies on a bending line. If $S_{\Omega}$ is finitely-bent, we define a measure on embedded intervals $\gamma$ in $S_{\Omega}$ by setting $\beta(\gamma)$ to be the sum of the bending encountered along $\gamma$. For more general domains, we define $\beta(\gamma)$ as an infimum over finitely-bent approximations between the endpoints of $\gamma$ (see [12] for details). Here, we are interested in the case where $\partial \Omega$ is smooth. In this case, the bending measure has a Radon-Nikodym derivative with respect to arc length on transverse intervals on the dome.
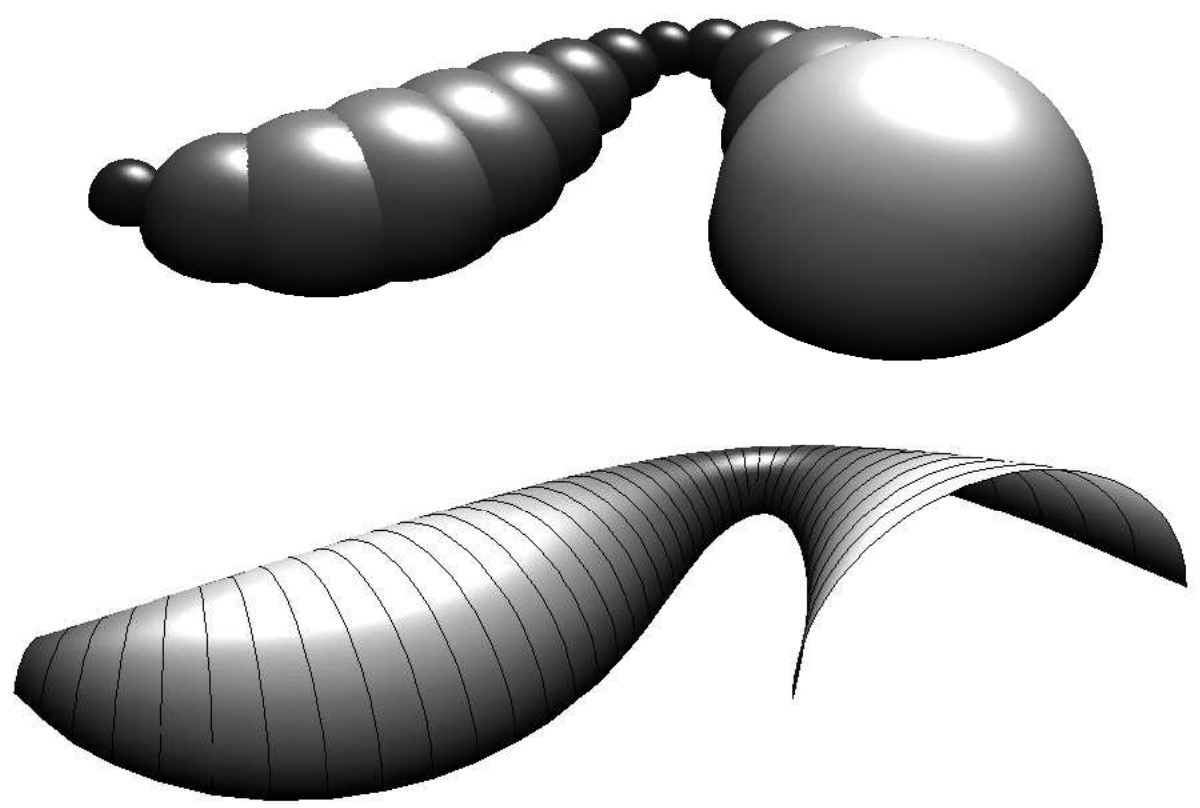

Figure 2. Above: $\Omega$ is a finite union of disks, shown in the half-space model. The faces of the dome meet at geodesic "bending lines", each with some bending angle $\beta$. Below: After refinement $S_{\Omega}$ is smooth; each support plane intersects $S_{\Omega}$ along a single bending line.

\section{Strip-like domains and the dome}

We now show that hyperbolic distances in $\Omega$ can be approximated by the same quantities on $S_{\Omega}$. This is advantageous as the Riemann map $\iota: S_{\Omega} \rightarrow \mathbf{D}$ from the dome to the unit disk admits an explicit construction which is easily understood in terms of the medial axis of $\Omega$ (see, for example, [7] or [18]). In particular, we exploit the fact that $\iota$ almost immediately gives extremal distances between pairs of bending lines: if $B_{s}$ and $B_{t}$, together with the arcs of $\partial \Omega$ joining their endpoints, bound a generalized quadrilateral $Q \in S_{\Omega}$, then up to a normalization by some map 
in $P S L_{2}(R)$, the map

$$
\log \left(\frac{1+\iota}{1-\iota}\right): S_{\Omega} \rightarrow\{(x, y):-\infty<x<\infty,-\pi / 2<y<\pi / 2\}
$$

takes $Q$ to a rectangle in the strip (see Figure 3 ).

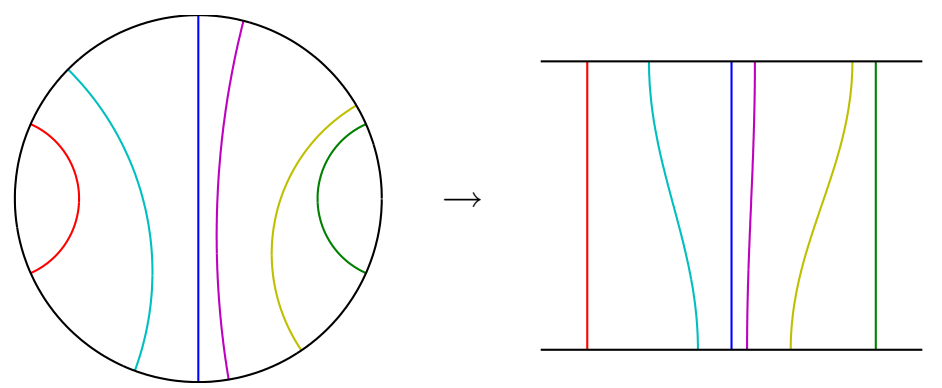

Figure 3. $\iota$ takes bending lines in the dome to geodesics in the disk. After mapping the disk to the strip, we immediately obtain hyperbolic distance $d_{h}$ and extremal distance $d_{S_{\Omega}}$ between bending lines $B_{s}$ and $B_{t}$ : that is, $d_{S_{\Omega}}\left(B_{s}, B_{t}\right)=\frac{1}{\pi} d_{h}\left(B_{s}, B_{t}\right)$.

3.1. Preliminaries: distance between bending lines. Our goal in this section is to obtain estimates for distances between pairs of bending lines in $S_{\Omega}$. We will later use this estimate to obtain explicit formulas for distances in $\Omega$ and to understand geometrically the length distortion introduced by the nearest-point retraction. We will require some basic definitions and a few results from [18]; we repeat them here without proof. First, the cross-ratio of 4 points in the complex plane can have 6 possible values depending on the order of the 4 points; we will use the following choice.

Definition 5. (Cross-ratio) Let $(a, b, c, d)$ be 4 points in the complex plane. Their cross-ratio is defined to be

$$
\chi(a, b, c, d)=\frac{(b-a)}{(d-a)} \frac{(d-c)}{(b-c)} .
$$

The next definition introduces a particular curve which is useful when estimating geodesic lengths in $S_{\Omega}$.

Definition 6. (Path of nearest points) Define the path of "nearest points"

$$
p^{*}(t)=\lim _{\epsilon \backslash t} \arg \min _{p \in B_{t}} d_{h}\left(p, B_{t+\epsilon}\right)
$$

and the infinitesimal distance between them

$$
d^{*}(t)=\lim _{\epsilon \rightarrow 0} \frac{1}{\epsilon} d_{h}\left(B_{t}, B_{t+\epsilon}\right) .
$$

We have the following formula for the infinitesimal cross-ratio of endpoints of adjacent bending lines; this formula will allow us to pass from statements about hyperbolic distance in $S_{\Omega}$ to explicit formulas involving the geometry of $\partial \Omega$.

Proposition 1. (Cross-ratio of endpoints) Let $a, b$ be the endpoints of some bending line at $t=0$. Then the infinitesimal cross-ratio $\chi_{\text {inf }}(t)$ satisfies

$$
\chi_{i n f}(t) \equiv \lim _{\epsilon \searrow 0} \frac{1}{t^{2}} \chi\left(a_{t}, a_{t+\epsilon}, b_{t+\epsilon}, b_{t}\right)=-\frac{|\dot{a} \dot{b}|}{|b-a|^{2}},
$$


where $\dot{a}=\left.\frac{d a}{d t}\right|_{t}$, similar for $\dot{b}$.

The next lemma is stated in non-invariant terms; it connects the Euclidean geometry of $\Omega$ to invariant quantities. We make the following definition:

Definition 7. (Midpoint of $B_{t}$ ) Fix a chart for the half-space model of $\mathbf{H}^{3}$. Then the midpoint of a bending line $B_{t}$ for a given domain $\Omega \subset \hat{\mathbf{C}}$ is the midpoint of $B_{t}$ viewed a Euclidean semicircle in this chart.

The next three lemmas describe $p^{*}(t)$ with explicit formulas.

Lemma 1. (Location of $p^{*}$ ) Let $a, b \in \partial \Omega$ be the endpoints of some bending line $B_{0}$. Then $p^{*}$ lies at hyperbolic distance $\frac{1}{2} \log \left|\frac{\dot{a}}{\dot{b}}\right|$ from the midpoint of $B_{0}$. Positive distances indicate motion towards $b$.

Lemma 2. (Infinitesimal distance between bending lines)

$$
d^{*}(t)=2 \frac{\sqrt{|\dot{a} \dot{b}|}}{|b-a|} .
$$

Lemma 3. The velocity $\dot{p}^{*}(t)$ decomposes into components $\dot{p}_{T}^{*}(t), \dot{p}_{\perp}^{*}(t)$, tangential and orthogonal to the bending line $B_{t}$ respectively, with hyperbolic lengths

$$
\begin{aligned}
\left\|\dot{p}_{T}^{*}\right\|_{h} & =\frac{|\dot{a}|-|\dot{b}|}{|b-a|} \dot{R}+\frac{1}{2}\left(\frac{\ddot{b}}{\dot{b}}-\frac{\ddot{a}}{\dot{a}}\right), \\
\left\|\dot{p}_{\perp}^{*}\right\|_{h} & =2 \frac{\sqrt{|\dot{a} \dot{b}|}}{|b-a|} .
\end{aligned}
$$

Further, $p^{*}$ meets $B_{t}$ at angle

$$
\theta(t)=\arctan \left(\frac{d^{*}(t)}{\left\|\dot{p}_{T}^{*}\right\|}\right)
$$

and has hyperbolic speed

$$
\left\|\dot{p}^{*}\right\|_{h}=\left(\left\|\dot{p}_{T}^{*}\right\|_{h}^{2}+\left\|\dot{p}_{\perp}^{*}\right\|_{h}^{2}\right)^{1 / 2}
$$

Remark. One may also write the tangential component as

$$
\left\|\dot{p}_{T}^{*}\right\|_{h}=\kappa_{m} \frac{\dot{R}}{\sqrt{1-\dot{R}^{2}}}+\frac{\partial}{\partial t} \frac{1}{2} \log \left|\frac{\dot{b}}{\dot{a}}\right| .
$$

While more cluttered, it does allow one to see at a glance the role played by the curvature of the medial axis.

The boundary derivatives of the map $\iota: \partial \Omega \rightarrow \partial D_{T}$ are particularly simple:

Proposition 2. Let $\iota: S_{\Omega} \rightarrow D_{T}$ map $S_{\Omega}$ to some medial disk $D_{T}$. Then $\iota$ is differentiable with

$$
\left|\frac{d \iota}{d s}\right|=1
$$

at iota's fixed points $a(T)$ and $b(T)$, where $s$ is the arclength parameter along $\partial \Omega$. 
Remark. This easy but useful result is essentially just the observation that $\iota$ is a rotation near the fixed points. It also highlights a key difference between the $\iota$ and Riemann maps: the derivatives of the Riemann map are hugely influenced by boundary curvature; the derivatives of $\iota$ are completely blind to curvature.

The next lemma is the main goal of this section. It gives us estimates for distances between bending lines along a branch of the medial axis. In upcoming sections we will generate more abstract estimates and then plug in Lemma 4 to obtain computable results.

Lemma 4. (Length of geodesics in $S_{\Omega}$ ) Let $\gamma \subset S_{\Omega}$ be the shortest geodesic joining some $B_{s}$ and $B_{t}, s<t$. Then

$$
2 \int_{s}^{t} \frac{\sqrt{|\dot{a} \dot{b}|}}{|b-a|} d u \leq L_{h}[\gamma] \leq 2 \int_{s}^{t} \sqrt{\frac{|\dot{a} \dot{b}|}{|b-a|^{2}}+\frac{1}{4}\left(\frac{|\dot{a}|-|\dot{b}|}{|b-a|} \dot{R}+\frac{1}{2}\left(\frac{\ddot{b}}{\dot{b}}-\frac{\ddot{a}}{\dot{a}}\right)\right)^{2}} d u
$$

whenever the curve $p^{*}$ is continuous.

Proof. Choose any path $p(u)$ joining $B_{s}$ and $B_{t}$. The lower bound is simply the observation that

$$
\|\dot{p}(u)\|_{h} \equiv \lim _{\epsilon \rightarrow 0} \frac{1}{\epsilon}\|p(u+\epsilon)-p(u)\|_{h} \geq \lim _{\epsilon \rightarrow 0} \frac{1}{\epsilon} d_{h}\left(B_{u}, B_{u+\epsilon}\right) \equiv d^{*}(u)
$$

for any choice of path $p(u)$.

The upper bound is obtained by integrating $\left\|\dot{p}^{*}\right\|_{h}$ along the path $p^{*}(u)$; the formula follows directly from Lemma 3 .

Remark. This estimate is sharp, which implies the path $p^{*}(u)$ is the geodesic joining $B_{s}$ and $B_{t}$, when $\left\|\dot{p}_{T}^{*}\right\|_{h}=0$. This can happen, for example, when $\frac{d}{d t} \log \sqrt{\frac{|\dot{b}|}{|\dot{a}|}}=$ 0 and either $\kappa_{m}$ or $\dot{R}$ is zero. The first happens exactly when $|\dot{b} / \dot{a}|$ is constant, or using some facts from the medial axis,

$$
\frac{\dot{b}}{\dot{a}}=\frac{1-R \kappa_{a}}{1-R \kappa_{b}}=\text { const. }
$$

Both conditions are satisfied in, for example, a strip-like domain consisting of two curves symmetric about a straight axis, or a section of an annulus. Note that $p^{*}$ can be geodesic without minimizing distance between any pair of bending lines and with $\dot{p}_{T}^{*} \neq 0$. The geodesic joining 0 to $\infty$ in the complement of a logarithmic spiral is such an example.

3.2. Hyperbolic distances in $S_{\Omega}$ and $\Omega$. We now obtain estimates relating hyperbolic distances in $\Omega$ and $S_{\Omega}$. We first bound the hyperbolic metric in $\Omega$ using the metrics on the maximal disks $\left\{D_{t}\right\}$. In a domain with real-analytic boundary, the medial axis is finitely-branched; the foliation consists of sets of $\operatorname{arcs}\left\{F_{t}\right\}$ along each branch. Where 3 or more branches meet, the foliation has a gap, which is an ideal polygon in some maximal disk $D$. The boundary of the polygon consists of ideal geodesics in $D$ with endpoints at the points of tangency of $D$. The next lemma is a variant of the Koebe $1 / 4$ theorem and allows us to control hyperbolic distance in $\Omega$ using the metric on the maximal disks. 
Lemma 5. Let $\Omega$ be a domain with real-analytic boundary. Then for $z \in \Omega$

$$
\frac{1}{2} \rho_{D}(z) \leq \rho_{\Omega}(z) \leq \rho_{D}(z)
$$

where $D$ is the medial disk which corresponds to the foliating arc or gap containing $z$, and $\rho_{\Omega}$ and $\rho_{D_{t}}$ denote hyperbolic arc length on $\Omega$ and $D$, respectively.

Proof. The upper bound follows from extension of domain, so we need only prove the lower bound. We first consider the case where $z$ lies on some foliating arc $F$. It is sufficient to consider the case where $z=0, D$ is the unit disk, and $F=[-1,1]$. Let $\sigma: \mathbf{C} \rightarrow \mathbf{C}$ be the map $\sigma(z)=\frac{1+z}{1-z}$. Let $f: D \rightarrow \Omega$ with $f(0)=0$. Then by the Koebe $1 / 4$ theorem,

$$
4 \geq\left|(\sigma \circ f)^{\prime}(0)\right|=2\left|f^{\prime}(0)\right| .
$$

Since conformal maps are hyperbolic isometries, we have

$$
\frac{\rho_{D}(0)}{\rho_{\Omega}((\phi \circ f)(0))}=\left|f^{\prime}(0)\right| \leq 2 \text {. }
$$

Now assume that $z$ lies in some gap. We need only consider the case where the gap is a triangle (every point in an n-gon is also contained in a triangle joining some triple of endpoints) and $z=0$. In this case, we know $\frac{\rho_{D}}{\rho_{\Omega}} \leq 2$ on each foliating arc on the boundary of the gap. In fact, $\frac{\rho_{D}}{\rho_{\Omega}}$ is a subharmonic function (see [17]) and achieves its maximum on the boundary, so the conclusion follows.

This is in fact sufficient to show that (2) holds in any domain, since we may approximate an arbitrary domain with a real-analytic domain, say, by considering the image of the circle of radius $r<1$ under a conformal map from the unit disk to the domain. The hyperbolic distances will converge as we let $r \rightarrow 1$ and the inequalities will still hold in the limit.

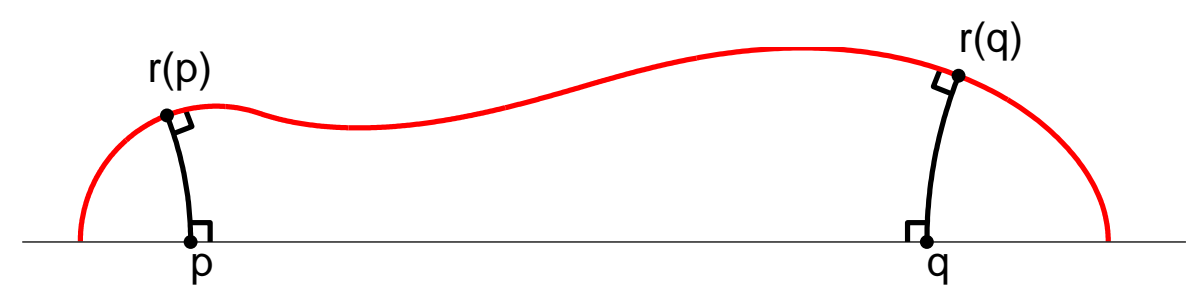

Figure 4. The situation for Theorem 1. One imagines the domain $\Omega$ lying in the plane with the dome above; we see this here in cross-section with the points $p, q$ mapped to the dome via the nearest-point retraction.

We can now apply Lemma 5 to prove our first theorem relating hyperbolic arclength in $S_{\Omega}$ to arclength in $\Omega$.

Theorem 1. (Hyperbolic distances comparable) Let $\gamma:[0, T] \rightarrow \Omega$ be path in $\Omega$ and $\sigma=r(\gamma)$. Then the hyperbolic velocities of $\gamma$ and $\sigma$ satisfy

$$
\frac{1}{2}\|\dot{\sigma}\|_{S_{\Omega}} \frac{1+\kappa_{1}}{1+\kappa_{1} \sin \theta} \leq\|\dot{\gamma}\|_{\Omega} \leq\|\dot{\sigma}\|_{S_{\Omega}}\left(1+\kappa_{1} \cos \theta\right)
$$

where $\theta$ is the angle between $\dot{\sigma}$ and the transverse vector to the bending line on which $\sigma$ lies, $\kappa_{1}$ is the hyperbolic principal curvature of $S_{\Omega}$, and the norms $\|\cdot\|_{\Omega}$ and $\|\cdot\|_{S_{\Omega}}$ denote hyperbolic length on $\Omega$ and $S_{\Omega}$. 
Proof. By conformal invariance, we need only consider a single point where $\gamma=0$ with velocity $\dot{\gamma}=\frac{\partial}{\partial t} \gamma$, the maximal circle is the unit circle, and the points of tangency are $a=-i, b=i$. Hence $r(\gamma)=(0,0,1)$. In this case, we have

$$
1 \leq \rho_{\Omega}(\gamma) \leq 2
$$

by Lemma 5 , and $\rho_{S_{\Omega}}(r(\gamma))=1$. Let $\dot{\sigma}=r_{*} \dot{\gamma}$ and in transverse coordinates on $\Omega$ and $S_{\Omega}$ write

$$
\dot{\gamma}=(|\dot{\gamma}| \cos \theta,|\dot{\gamma}| \sin \theta), \quad \dot{\sigma}=(|\dot{\sigma}| \cos \theta,|\dot{\sigma}| \sin \theta) .
$$

We evaluate the upper bound

$$
\begin{aligned}
\|\dot{\gamma}\|_{\Omega} & =\rho_{\Omega}\left|J^{-1} \dot{\sigma}\right| \leq 2 \sqrt{\left(\frac{\dot{\sigma}_{1}}{2}\right)^{2}\left(1+\kappa_{1}\right)^{2}+\left(\frac{\dot{\sigma}_{2}}{2}\right)^{2}} \\
& =\sqrt{|\dot{\sigma}|^{2}+\dot{\sigma}_{1}^{2}\left(2 \kappa_{1}+\kappa_{1}^{2}\right)}=|\dot{\sigma}| \sqrt{1+\cos ^{2} \theta\left(2 \kappa_{1}+\kappa_{1}^{2}\right)} .
\end{aligned}
$$

We now need the inequality $\sqrt{1+a^{2}\left(2 b+b^{2}\right)} \leq 1+a b$, for $0 \leq a<1, b>0$. Indeed,

$$
(1+a b)^{2}=1+2 a b+a^{2} b^{2} \geq 1+2 a^{2} b+a^{2} b^{2} .
$$

Hence,

$$
\|\dot{\gamma}\|_{\Omega} \leq|\dot{\sigma}|\left(1+\kappa_{1} \cos \theta\right)=\|\dot{\sigma}\|_{S_{\Omega}}\left(1+\kappa_{1} \cos \theta\right) .
$$

We now address the lower bound:

$$
\begin{aligned}
\|\dot{\sigma}\|_{S_{\Omega}} & =\rho_{S_{\Omega}}|J \dot{\gamma}|=\sqrt{\left(\frac{2 \dot{\gamma}_{1}}{1+\kappa_{1}}\right)^{2}+\left(2 \dot{\gamma}_{2}\right)^{2}} \\
& =\frac{2}{1+\kappa_{1}} \sqrt{\dot{\gamma}_{1}^{2}+\dot{\gamma}_{2}^{2}\left(1+\kappa_{1}\right)^{2}}=\frac{2|\dot{\gamma}|}{1+\kappa_{1}} \sqrt{1+\sin ^{2} \theta\left(2 \kappa_{1}+\kappa_{1}^{2}\right)} \\
& \leq \frac{2\|\dot{\gamma}\|_{\Omega}}{1+\kappa_{1}} \sqrt{1+\sin ^{2} \theta\left(2 \kappa_{1}+\kappa_{1}^{2}\right)} \leq 2\|\dot{\gamma}\|_{\Omega} \frac{1+\kappa_{1} \sin \theta}{1+\kappa_{1}} .
\end{aligned}
$$

Remark. Theorem 1 holds trivially by Lemma 5 at points where $\gamma$ lies in a gap.

The quantity $\kappa_{1} \cos \theta$ is the amount of bending encountered per unit hyperbolic length (see [18]) in the direction $\dot{\sigma}$. Thus we may write

$$
\|\dot{\gamma}\|_{\Omega} \leq\|\dot{\sigma}\|_{S_{\Omega}}+d \beta
$$

The lower bound admits a similar interpretation: $\kappa_{1}$ is again the bending per unit length in the transverse direction. If the velocity $\dot{\sigma}$ is transverse to the bending lines, then $\sin \theta=0$ and we have

$$
\frac{1}{2}\left(\|\dot{\sigma}\|_{S_{\Omega}}+d \beta\right) \leq\|\dot{\gamma}\|_{\Omega}
$$

whereas when $\dot{\sigma}$ is parallel to the bending line, $\sigma$ encounters no bending and

$$
\frac{1}{2}\|\dot{\sigma}\|_{S_{\Omega}} \leq\|\dot{\gamma}\|_{\Omega}
$$

The integrated form of this observation is the following corollary relating path lengths in $S_{\Omega}$ and $\Omega$. 
Corollary 5. Let $\sigma:[0, T] \rightarrow S_{\Omega}$ be a transverse arc. Then

$$
\frac{1}{2}\left(L_{h}[\sigma]+\int_{\sigma} d \beta\right) \leq L_{h}[\gamma] \leq L_{h}[\sigma]+\int_{\sigma} d \beta
$$

where $\gamma=r^{-1}(\sigma)$.

Proof. As we remarked, the upper bound holds in general. By Theorem 10, we have

$$
\|\dot{\gamma}\|_{\Omega} \geq \frac{1}{2}\|\dot{\sigma}\|_{S_{\Omega}} \frac{1+\kappa_{1}}{1+\kappa_{1} \sin \theta}=\frac{1}{2}\|\dot{\sigma}\|_{S_{\Omega}}\left(1+\kappa_{1}\right)=\frac{1}{2}\left(\|\dot{\sigma}\|_{S_{\Omega}}+d \beta\right)
$$

where the first equality uses the fact that $\sigma$ is a transverse arc implies $\theta \equiv 0$. The corollary follows by integration.

Remark. Lurking behind the estimates on hyperbolic distances is a nice interpretation of crescents in the finitely-bent case. The previous corollary showed that up to constants, hyperbolic lengths of transverse arcs agreed up to the amount of bending encountered along the path. In the finitely-bent case, one can view the crescents (the preimages of bending lines under $r$ ) as ideal strips of approximately constant hyperbolic width. We present a proof in the finitely-bent case.

Lemma 6. (Width of crescents) In a finitely-bent domain, let $B \subset S_{\Omega}$ be a bending line with bending angle $\beta$ and let $C \subset \Omega$ be the associated crescent $r^{-1}(B)$. Let $F^{-}$and $F^{+}$be the two circular arcs bounding $C$. Then

$$
\frac{1}{2} \beta \leq d_{h}\left(F^{-}, F^{+}\right) \leq \beta,
$$

where $d_{h}$ is hyperbolic distance in $\Omega$.

Proof. Let $a$ and $b$ be the two points in $\partial C \cap \partial \Omega$. We consider $C$ as a disjoint union of circular arcs $F_{\theta}, 0<\theta<\beta$, where $F_{\theta}$ joins $a$ to $b$ and meets $F^{-}$at angle $\theta$. By Lemma 5,

$$
\frac{1}{2} \rho_{D_{\theta}} \leq \rho_{\Omega} \leq \rho_{D_{\theta}}
$$

on $F_{\theta}$, where $\rho_{\Omega}$ defines the hyperbolic metric on $\Omega$ and $D_{\theta} \subset \Omega$ is the disk containing $F_{\theta}$ as an ideal geodesic in the Poincaré metric $\rho_{D_{\theta}}$. Let $\sigma$ be any Möbius map taking $a$ to 0 and $b$ to $\infty$. Then $\sigma(C)$ is a sector of angular width $\beta$ and $\sigma\left(F_{\theta}\right)$ is a straight line extending from 0 to $\infty$. For any $\theta$, the pushforward under $\sigma$ of $\rho_{D_{\theta}}$ is simply the metric given by $1 / r$ in polar coordinates, and we see that

$$
\frac{1}{2 r} \leq \sigma_{*} \rho_{\Omega} \leq \frac{1}{r}
$$

If we show that arcs of circles centered at 0 are geodesic in the metric $1 / r$, the conclusion will follow. This is indeed the case: Under the map $\phi(z)=\log (z)$, the sector maps to a strip and the metric $\rho=1 / r$ pushes forward to the metric on the strip given by

$$
\left(\phi_{*} \rho\right)(w) \equiv \frac{\rho\left(\phi^{-1}(w)\right)}{\left|\phi^{\prime} \circ \phi^{-1}(w)\right|}=\frac{1}{e^{w}} \cdot e^{w}=1 .
$$

Thus any two points on the boundary of the strip are joined by the Euclidean straight line between them, the shortest such paths have length $\beta$, and the conclusion follows. 
3.3. Best Lipschitz constant for $r: \Omega \rightarrow S_{\Omega}$. The lower estimates in Theorem 1 and Corollary 5 provide a refinement of the Thurston-Sullivan " $L=2$ " conjecture. This conjecture arose in the study of hyperbolic 3-manifolds, and was originally worked on in the 80's by Epstein and Marden, who showed that $L<4$ (see [15]). A series of improvements, including work by Bridgeman, Canary, and Bishop, followed, progressively lowering the best constant (see, e.g., [10, 11, 9]). The best possible constant $L=2$ was finally proven by Epstein, Marden and Markovic in [17]. We obtain their result here working with domains with real-analytic boundaries, instead of finitely-bent domains. Our result is the following.

Theorem 2. (" $L=2$ " conjecture) The nearest point retraction map $r: \Omega \rightarrow S_{\Omega}$ is 2-Lipschitz in the hyperbolic metrics. Further, if $\gamma \in \Omega$ is a path whose retract $\sigma=r(\gamma)$ is transverse to any bending lines it encounters, we have

$$
L_{h}[\sigma] \leq 2 L_{h}[\gamma]-\int_{\sigma} d \beta
$$

Proof. Along any branch of the medial axis,

$$
\|\dot{\sigma}\|_{S_{\Omega}} \leq 2\|\dot{\gamma}\|_{\Omega} \frac{1+\kappa_{1} \sin \theta}{1+\kappa_{1}}
$$

The right-hand side is less than $2\|\dot{\gamma}\|_{\Omega}$ in general, which proves the 2-Lipschitz statement along any branch of the axis. If $\dot{\sigma}$ is transverse to the bending lines on some branch, then $\sin \theta=0$ and

$$
\|\dot{\sigma}\|_{S_{\Omega}} \leq \frac{2}{1+\kappa_{1}}\|\dot{\gamma}\|_{\Omega}
$$

which proves the second statement after integrating. On any gap, both statements follow easily from Lemma 5 and the fact that curvature is zero.

This is sufficient to prove the result on a domain with real-analytic boundary, since the medial axis of such a domain is finitely-branched. In general, we approximate any domain $\Omega$ with a sequence of domains $\Omega_{r}$ bounded by the (real-analytic) image of the circle of radius $r<1$ under a conformal map from the unit disk to $\Omega$. For sufficiently large $r$, any pair of points $p, q \in \Omega$ are in the subdomain $\Omega_{r}$, and hyperbolic distances converge in $\Omega_{r}$ and $S_{\Omega_{r}}$ to hyperbolic distances in $\Omega$ and $S_{\Omega}$. The inequalities then hold in the limit.

The 2-Lipschitz result is sharp in the case of a "folded" surface. For example, take $\Omega$ to be the complement in the complex plane of the negative part of the real axis. The dome of this domain consists of two half planes, glued together. This dome has only one bending line. This is the extremal domain for the Koebe $1 / 4$ theorem and the hyperbolic metric in $\Omega$ is exactly $1 / 2$ at $z=1$. It is simple to show that in this example the nearest point retraction is 2-Lipschitz.

In general, the metrics on $\Omega$ and $S_{\Omega}$ are comparable up to a factor of 2 , while the more bent the dome is, the smaller the transverse part of the Jacobian of $r$. From this point of view, the folded surface is extremal because it makes the hyperbolic metric in $S_{\Omega}$ as large as possible relative to $\Omega$ while simultaneously concentrating the bending on a single line. In this case, $r$ can't help "slow down" the image of a particle following the path $\gamma$ as it approaches the bending line. 


\section{Extremal distances in $S_{\Omega}$ and $\Omega$}

In this section, our goal is to relate extremal distances in $S_{\Omega}$ to those in $\Omega$, with explicit estimates involving the geometry of $\partial \Omega$. We first record a short lemma showing that estimates for for extremal and hyperbolic distances are interchangeable up to a constant. We then prove the main theorem of this section, which shows that extremal distances between foliating $\operatorname{arcs} F_{s}, F_{t}$ in $\Omega$ agree with the corresponding distances between their retracts $B_{s}, B_{t}$ in $S_{\Omega}$, up to an error which is controlled by the bending of $S_{\Omega}$. From these two results, it is easy to obtain some explicit formulas for extremal distances using material from the previous sections. The final estimate takes the form of a variant of the Ahlfors distortion theorem, in which we replace an integral over a strip domain with a similar integral over any branch of the medial axis; this integral provides bounds on extremal distances in $S_{\Omega}$ and also on $\Omega$. We now prove the first lemma.

Lemma 7. (Extremal and hyperbolic distances coincide for $\left\{B_{t}\right\}$ ) Let $B_{s}, B_{t} \subset$ $S_{\Omega}, s<t$, be two bending lines. Then the extremal distance $d_{S_{\Omega}}$ and the hyperbolic distance $d_{h}$ satisfy

$$
d_{S_{\Omega}}\left(B_{s}, B_{t}\right)=\frac{1}{\pi} d_{h}\left(B_{s}, B_{t}\right)
$$

Proof. The $\iota$ map takes $B_{s}$ and $B_{t}$ to a pair of geodesics $\iota\left(B_{s}\right), \iota\left(B_{t}\right)$ in the disk. Then for some angle $0<\phi<\pi / 2$, there is a map $\sigma: \mathbf{D} \rightarrow \mathbf{D}$ taking the endpoints of $\iota\left(B_{t}\right)$ to $\left\{e^{-i \phi}, e^{i \phi}\right\}$ and the endpoints of $\iota\left(B_{s}\right)$ to $\left\{e^{i(\pi-\phi)}, e^{-i(\pi-\phi)}\right\}$. For such a $\sigma$, the map $z \rightarrow \log \frac{1+\sigma(z)}{1-\sigma(z)}$ takes the disk to the strip $\{x+i y:-\pi / 2<y<\pi / 2\}$ and takes $\iota\left(B_{s}\right)$ and $\iota\left(B_{t}\right)$ to the vertical sides of a rectangle of height $\pi$. Since the map is an isometry between the hyperbolic metrics on the disk and the strip, the proof is complete.

We now proceed to the main theorem and show that extremal distances in $\Omega$ can be approximated by those in $S_{\Omega}$. This is nice because the relatively simple geometry of $S_{\Omega}$ allows explicit estimates, as recorded in earlier sections.

Theorem 3. Let $F_{s}, F_{t} \subset \Omega$ be two foliating arcs. Then

$$
d_{S_{\Omega}}\left(B_{s}, B_{t}\right) \leq d_{\Omega}\left(F_{s}, F_{t}\right) \leq d_{S_{\Omega}}\left(B_{s}, B_{t}\right)+\frac{2}{\pi^{2}} \int_{s}^{t} d \beta
$$

where $\beta(t)$ is the bending measure associated with $S_{\Omega}$.

Proof. We appeal to the the finitely-bent case. Let $\rho_{S}$ be the extremal metric for $S_{\Omega}$. Observe that the nearest-point retraction $r^{-1}$ maps each facet of the dome conformally onto its image in $\Omega$. Define the metric $\rho_{\Omega}$ on $\Omega$ to be $r^{*} \rho_{S}$ (the pullback under the nearest-point retraction), where this is defined, and let $\rho_{\Omega}=0$ elsewhere. This metric achieves the lower bound.

We explicitly construct a metric to produce the upper bound. Consider two bending lines $B_{j}$ and $B_{k}, k>j$. The region of the dome between these lines is a generalized quadrilateral, and there is some extremal map taking this quadrilateral to a rectangle. In fact, this map is simply $\iota$ followed by the transformation $z \rightarrow \log \frac{1+z}{1-z}$ and a normalizing conformal self-map of the strip. Hence we see that the extremal map takes bending lines to geodesics in the strip model. 
Let $\phi_{n}$ be the restriction of this extremal map to the $n$-th facet of the dome; $\phi_{n}$ takes the facet to a region in the strip bounded by a pair of geodesics. The map $\phi_{n} \circ r$ takes a subset of each medial disk in $\Omega$ to the strip.

Now let $C_{n}$ be the crescent between faces $n$ and $n+1$ and note that on each bending line, $\phi_{n}$ and $\phi_{n+1}$ agree. On $C_{n}$, define coordinates $0<r<\infty, 0<\theta<\beta$ by sending the two endpoints of the crescent to 0 and $\infty$ and the center to (say) 1 , and taking standard polar coordinates in this frame. The map

$$
\sigma_{n}(z)=\left(\phi_{n} \circ r\right)(z)+\theta(z)
$$

takes $C_{n}$ to a region of the strip bounded by two geodesics; $\sigma_{n}$ agrees with $\phi_{n}$ and $\phi_{n+1}$ on the boundary, up to translation. One may verify that the map $\sigma_{n}$ is equivalent to the map

$$
r e^{i \theta} \mapsto \theta+i \arctan r
$$

taking the crescent to a rectangle of height $\pi$ and width $\beta$, followed by a self-map of the strip applied separately to each vertical line in the rectangle.

Now let $R$ be a rectangle of height $\pi$ and width $\pi^{2} d_{S_{\Omega}}\left(B_{s}, B_{t}\right)+\sum_{n} \beta(n)$, and define the continuous map $\Psi: \Omega \rightarrow R$ by gluing together the images of adjacent maps $\phi_{1}, \sigma_{1}, \phi_{2}, \sigma_{2}, \ldots, \phi_{n}$ along the sides of length $\pi$.

Define the metric $\rho_{\Omega}$ on $R$ to be $|\nabla \operatorname{Im} \Psi|$. Clearly the length $L_{\rho_{\Omega}}$ of any path joining the top and bottom of the rectangle is at least $\pi$. We need an upper bound on the area. Since this metric is the extremal metric on each face of the dome, we have that the combined area of all faces is $\pi^{2} d_{S_{\Omega}}\left(B_{j}, B_{k}\right)$.

On each crescent $C_{n}$, the map $\Psi$ is given by $(r, \theta) \mapsto \theta+i \arctan r$ followed by a self-map of the strip applied to each vertical line. If the self-map is the identity, we have

$$
\begin{aligned}
A_{\rho_{\Omega}}\left(C_{n}\right) & =\int_{0}^{\beta(n)} \int_{0}^{\infty}|\nabla \operatorname{Im} \Psi|^{2} r d r d \theta=\int_{0}^{\beta(n)} \int_{0}^{\infty} \frac{r}{\left(1+r^{2}\right)^{2}} d r d \theta \\
& =-\left.\beta(n) \frac{1}{2\left(1+r^{2}\right)}\right|_{0} ^{\infty}=\frac{\beta(n)}{2} .
\end{aligned}
$$

It remains to be shown that area is nonincreasing when the self-map is other than the identity. We need to evaluate the area of a sector under the metric $d s^{2}=$ $|\nabla \operatorname{Im} \Psi|^{2}\left(d x^{2}+d y^{2}\right)$, that is

$$
\int_{0}^{\beta} \int_{0}^{\infty}|\nabla \operatorname{Im} \Psi|^{2} r d r d \theta
$$

where

$$
\Psi(r, \theta)=\sigma(i(2 \arctan r-\pi / 2))+\theta
$$

for some conformal self-map $\sigma$ of the strip. We claim we need only consider $\sigma$ which are purely elliptic with fixed point 0 . We can decompose an arbitrary element $\sigma \in P S L_{2}(\mathbf{R})$ into a choice of $\sigma(0)$ and an elliptic rotation about $\sigma(0)$. Moving $\sigma(0)$ can be decomposed as a pair of hyperbolics, first with axis equal to the $\mathrm{x}$-axis and the second with axis perpendicular. The first is simply translation and the metric is obviously invariant. In the second case, the action of $\sigma$ pulls back to a conformal 
self-map of the sector: it is the restriction to the wedge of a hyperbolic $h: \hat{\mathbf{C}} \rightarrow \hat{\mathbf{C}}$ with fixed points at 0 and $\infty$. In this case

$$
\begin{aligned}
\iint_{S_{\beta}}|\nabla \operatorname{Im} \Psi(r, \theta)|^{2} & =\iint_{S_{\beta}}\left|\operatorname{Im} \frac{\partial}{\partial r} \sigma(i(2 \arctan r-\pi / 2))\right|^{2} \\
& =\iint_{S_{\beta}}\left|\operatorname{Im} \frac{\partial}{\partial r} i(2 \arctan h(r)-\pi / 2)\right|^{2} \\
& =\iint_{S_{\beta}}\left|\frac{d h}{d z}\right|^{2}\left|2 \arctan ^{\prime}(h(r))\right|^{2} \\
& =\iint_{h\left(S_{\beta}\right)}\left|2 \arctan ^{\prime}(r)\right|^{2}=\iint_{S_{\beta}}\left|2 \arctan ^{\prime}(r)\right|^{2}
\end{aligned}
$$

as claimed. Hence we need only consider elliptics. An elliptic $\sigma \in P S L_{2}(\mathbf{R})$ with rotation angle $\phi$ and fixed point at 0 acts on the strip as

$$
\sigma(z)=\log \frac{e^{z}+1+\alpha\left(e^{z}-1\right)}{e^{z}+1-\alpha\left(e^{z}-1\right)}
$$

for $\alpha=e^{i \phi}$. Further,

$$
\frac{d \sigma}{d z}=\frac{4 \alpha e^{z}}{\left(e^{2}+1\right)^{2}-\alpha^{2}\left(e^{z-1)^{2}}\right.}
$$

From this, one may compute

$$
|\nabla \operatorname{Im} \sigma(z)|_{z=i y}=\frac{d}{d y} \arctan (\cos \phi \tan y)=\frac{\cos \phi}{\cos ^{2} y+\cos ^{2} \phi \sin ^{2} y} .
$$

We now compute the area:

$$
\begin{aligned}
\iint_{S_{\beta}}|\nabla \operatorname{Im} \Psi(r, \theta)|^{2} & =\iint_{S_{\beta}}\left|\operatorname{Im} \frac{\partial}{\partial r} \sigma(i \arctan r)\right|^{2} \\
& =\int_{0}^{\beta} \int_{0}^{\infty}\left(\frac{\cos \phi}{\cos ^{2} y+\cos ^{2} \phi \sin ^{2} y} \frac{2}{1+r^{2}}\right)_{y=2 \arctan (r)-\frac{\pi}{2}}^{2} r d r d \theta
\end{aligned}
$$

A somewhat lengthy but direct computation shows that

$$
\iint_{S_{\beta}}|\nabla \operatorname{Im} \Psi(r, \theta)|^{2}=\beta \int_{-1}^{1}\left(\frac{\cos \phi}{1-v^{2} \sin ^{2} \phi}\right)^{2} d v .
$$

Differentiating under the integral sign followed by explicit integration reveals the extrema on $[0,2 \pi)$ are maxima at $\phi=0, \pi$ and minima at $\phi=\pi / 2,3 \pi / 2$. Therefore the conjugate extremal distance satisfies

$$
d_{\Omega}^{*}\left(F_{j}, F_{k}\right) \geq \frac{\pi^{2}}{\pi^{2} d_{S_{\Omega}}\left(B_{j}, B_{k}\right)+\frac{1}{2} \sum_{n} \beta(n)} .
$$

Hence

$$
d_{\Omega}\left(F_{j}, F_{k}\right) \leq d_{S_{\Omega}}\left(B_{j}, B_{k}\right)+\frac{2}{\pi^{2}} \sum_{n} \beta(n) .
$$

This completes the proof in the finitely-bent case; the smooth case follows under refinement. 
We can now show that on a regular branch of the medial axis there is a natural analog for the $\int \frac{d x}{\theta(x)}$ estimate for extremal distances in strip domains. This follows from the geodesic length estimates of Lemma 4, combined with the fact that extremal and hyperbolic distances between bending lines coincide. We make two corollaries to produce our Ahlfors integral. The first is an estimate for extremal distances in the dome.

Corollary 6. Let $B_{s}, B_{t}, s<t$, be two bending lines in $S_{\Omega}$. Then

$$
\frac{2}{\pi} \int_{s}^{t} \frac{\sqrt{\dot{a} \dot{b}}}{|b-a|} d u \leq d_{S_{\Omega}}\left(B_{s}, B_{t}\right) .
$$

Proof. By Lemmas 7 and 4, we have

$$
d_{S_{\Omega}}\left(B_{s}, B_{t}\right)=\frac{1}{\pi} d_{h}\left(B_{s}, B_{t}\right) \geq \frac{2}{\pi} \int_{s}^{t} \frac{\sqrt{\dot{a} \dot{b}}}{|b-a|} d u .
$$

Remark. This estimate is sharp when the geodesic joining $B_{t}$ and $B_{s}$ coincides with the axis of "nearest points", $p^{*}(u), s \leq u \leq t$.

The lower estimate applies without modification to the domain $\Omega$; this is our analog for the integral estimate $\int \frac{d x}{\theta}$ in strip domains.

Corollary 4. (A medial axis Ahlfors integral) Let $F_{s}, F_{t}, s<t$, be two foliating arcs in $\Omega$. Then

$$
d_{\Omega}\left(F_{s}, F_{t}\right) \geq \frac{2}{\pi} \int_{s}^{t} \frac{\sqrt{\dot{a} \dot{b}}}{|b-a|} d m .
$$

Proof. By Theorem 3,

$$
d_{\Omega}\left(F_{s}, F_{t}\right) \geq d_{S_{\Omega}}\left(B_{s}, B_{t}\right) .
$$

There is also an upper bound. It seems to be empirical fact that upper bounds for extremal distance are often unwieldy compared to lower bounds.

Corollary 7. Let $F_{s}, F_{t}, s<t$, be two foliating arcs in $\Omega$. Then

$$
d_{\Omega}\left(F_{s}, F_{t}\right) \leq \frac{2}{\pi} \int_{s}^{t} \sqrt{\frac{|\dot{a} \dot{b}|}{|b-a|^{2}}+\frac{1}{4}\left(\frac{|\dot{a}|-|\dot{b}|}{|b-a|} \dot{R}+\frac{1}{2}\left(\frac{\ddot{b}}{\dot{b}}-\frac{\ddot{a}}{\dot{a}}\right)\right)^{2}} d u+\frac{2}{\pi^{2}} \int_{s}^{t} d \beta .
$$

Proof. Apply Lemmas 7 and 4, and Theorem 3 as above.

\section{Appendix: Geometry of the medial axis}

Many of our results in the work above are stated in terms of the geometry of the medial axis; we collect the necessary formulas and results here. Throughout this paper we work on a single smooth branch of the medial axis of some domain with smooth boundary arcs. In general, the medial axis of any domain with real-analytic boundary is finitely-branched and consists of a finite number of real-analytic curves (see [13]); it then follows that the dome $S_{\Omega}$ over a real-analytic branch of the medial axis is itself smooth. However, over branch points of the medial axis, the dome will generally be no more than $C^{1}$ no matter how smooth the boundary arcs are. 


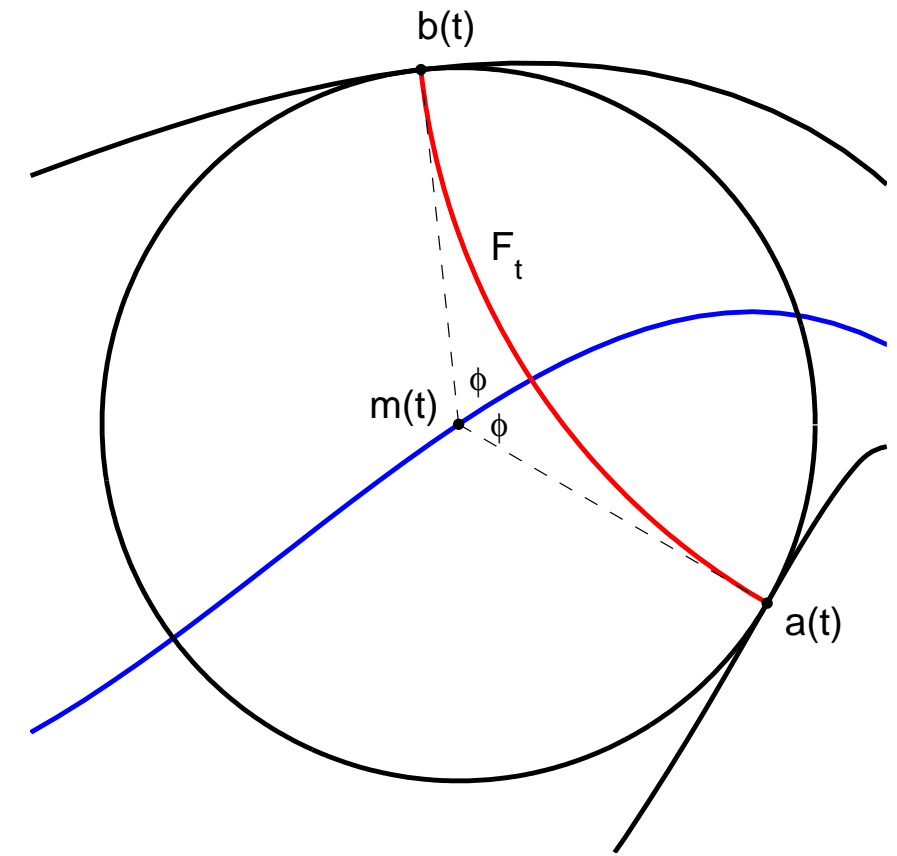

Figure 5. Notation for the medial axis. The axis is shown in blue; $m(t)$ is a parametrization of the medial axis. $a(t), b(t)$ are points of tangency. The red arc $F_{t}=r^{-1}\left(B_{t}\right)$ is the foliating arc connecting $a(t)$ and $b(t) . \phi=\arg (b-m)-\tau_{m}$ is the angle between one of the dashed lines and the tangent to the medial axis at $m$.

Definition 8. (Medial axis) The medial axis pair $(m(t), R(t))$ of a closed planar region consists of $m(t)$, the curve defined by the locus of centers of maximal circles contained in the region, and $R(t)$, their associated radii.

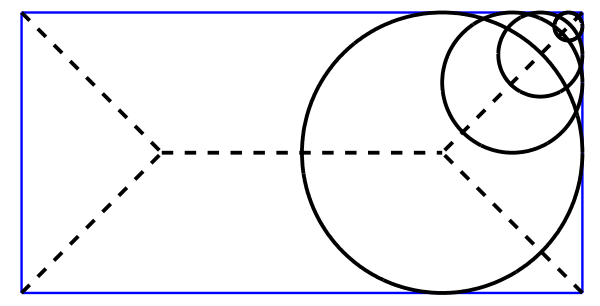

Figure 6. A rectangle and its medial axis. The branches of the axis are formed by the centers of maximal circles.

The curve $m(t)$ gives a sort of skeleton for a planar region. In general, $m$ can be quite pathological; for example, it may be infinitely branched, and may even have dimension $>1 .{ }^{1}$ However, for a region with real-analytic boundary, $m$ consists of a finite number of smooth branches [13] meeting at branch points.

Throughout we use the notation introduced above; see Figure 5. By a smooth branch of the medial axis, we mean a smooth, arclength-parametrized (unless stated otherwise) pair $(m(t), R(t))$ for a region with smooth boundary.

The following results can be found in [23]. We first show how to reconstruct the boundary given the medial axis pair.

${ }^{1}$ C. Bishop, personal communication. 
Theorem 8. Along a smooth branch of the medial axis:

(1) The boundary curves corresponding to $(m(t), R(t))$ are given by

$$
\begin{aligned}
& a(t)=\left(m+R \dot{R} e^{i \tau_{m}}-R \sqrt{1-\dot{R}^{2}} e^{i \nu_{m}}\right)(t), \\
& b(t)=\left(m+R \dot{R} e^{i \tau_{m}}+R \sqrt{1-\dot{R}^{2}} e^{i \nu_{m}}\right)(t) .
\end{aligned}
$$

(2) Given associated boundary points $a(s), b(s)$, the corresponding medial axis pair is given by

$$
R(s)=\frac{|b(s)-a(s)|}{2 \sin \phi(s)}, \quad m(s)=a(s)+R(s) e^{i \nu_{a}}, \quad=b(s)+R(s) e^{i \nu_{b}} .
$$

Not all pairs $(m(t), R(t))$ are allowable. The following theorem identifies those pairs which are.

Theorem 9. The smooth pair $(m(t), R(t))$ is locally the medial axis of a smooth boundary curve if and only if

(1) $|\dot{R}|<1$,

(2) $\left|\kappa_{m}\right|<\frac{1-\dot{R}^{2}-R \ddot{R}}{r \sqrt{1-\dot{R}^{2}}}$.

We will frequently need to use the following relationships. See [23] for various other relationships which hold along a smooth branch.

Proposition 3. Along a smooth branch of the medial axis,

$$
\begin{aligned}
\dot{R} & =-\cos \phi, \quad \frac{|b-a|}{2 R}=\sin \phi, \quad|\dot{a}|=\frac{\sqrt{1-\dot{R}^{2}}}{1-R \kappa_{a}} \\
|\dot{b}| & =\frac{\sqrt{1-\dot{R}^{2}}}{1-R \kappa_{b}}, \quad \kappa_{m}=\sqrt{1-\dot{R}^{2}}\left(\frac{\kappa_{a}-\kappa_{b}}{\left(1-R \kappa_{a}\right)\left(1-R \kappa_{b}\right)}\right) .
\end{aligned}
$$

We'll also need a few variations on these formulas; the next proposition gives a short proof of some relations we will use.

\section{Proposition 4.}

$$
\begin{aligned}
-\dot{\phi} & =\frac{1}{2}\left(\kappa_{a}|\dot{a}|+\kappa_{b}|\dot{b}|\right), \\
\kappa_{m} & =\frac{1}{2}\left(\kappa_{a}|\dot{a}|-\kappa_{b}|\dot{b}|\right)=\frac{|\dot{a}|-|\dot{b}|}{2 R} .
\end{aligned}
$$

Proof. At any time we have

$$
\nu_{b}=\tau_{m}+\theta_{b}+\pi, \quad \nu_{a}=\tau_{m}+\theta_{a}+\pi=\tau_{m}-\theta_{b}+\pi .
$$

According to our sign convention, $\kappa_{b}=-\frac{d \nu_{b}}{d t}$ and $\kappa_{a}=\frac{d \nu_{a}}{d t}$, so implicit differentiation yields

$$
\kappa_{b}|\dot{b}|=-\kappa_{m}|\dot{m}|-\dot{\theta}_{b}, \quad \kappa_{a}|\dot{a}|=\kappa_{m}|\dot{m}|-\dot{\theta}_{b}
$$


Formula (5) follows by adding these two equations; (6) follows by subtracting them. We continue computation to obtain the remaining results:

$$
\begin{aligned}
2 \kappa_{m}|\dot{m}| & =\kappa_{a}|\dot{a}|-\kappa_{b}|\dot{b}|, \\
2 \kappa_{m}|\dot{m}| & =\sqrt{1-\dot{R}^{2}}\left(\frac{\kappa_{a}}{1-R \kappa_{a}}-\frac{\kappa_{b}}{1-R \kappa_{b}}\right)=\sqrt{1-\dot{R}^{2}}\left(\frac{\kappa_{a}-\kappa_{b}}{\left(1-R \kappa_{a}\right)\left(1-R \kappa_{b}\right)}\right) \\
& =\frac{\sqrt{1-\dot{R}^{2}}}{R}\left(\frac{R \kappa_{a}-R \kappa_{b}}{\left(1-R \kappa_{a}\right)\left(1-R \kappa_{b}\right)}\right)=\frac{\sqrt{1-\dot{R}^{2}}}{R}\left(\frac{1}{1-R \kappa_{a}}-\frac{1}{1-R \kappa_{b}}\right) \\
& =\frac{1}{R}(|\dot{a}|-|\dot{b}|) .
\end{aligned}
$$

The formulas for reconstructing the boundary curve from the axis seem to indicate a derivative is lost when passing from one to the other. However, this is not the case. The following result appears in [23].

Theorem 10. Let $s, t$ and $v$ be arclength parameters for $a, b$ and $m$, respectively.

(1) If $(m(v), R(v))$ are a $C^{p}, p \geq 2$, interior portion of the medial axis pair for boundary curves $a, b$, with $|\dot{R}|<1$ and $\left|\kappa_{m}\right|<\frac{1-\dot{R}^{2}-R \ddot{R}}{r \sqrt{1-\dot{R}^{2}}}$, then $a$ and $b$ are also $C^{p}$.

(2) If $a(s), b(t)$ are portions of a $C^{p}$ curve, $p \geq 2$, corresponding via the medial axis so

$$
1-\kappa_{a b} \frac{|b-a|}{2 \sin \phi}>0
$$

the the associated interior portion of $m$ is $C^{p}$.

\section{References}

[1] Ahlfors, L. V.: Conformal invariants: topics in geometric function theory. - McGraw-Hill Series in Higher Mathematics, McGraw-Hill, New York, 1973.

[2] Ahlfors, L. V.: Complex analysis: an introduction to the theory of analytic functions of one complex variable. - International Series in Pure and Applied Mathematics, 3rd edition, McGraw-Hill, New York, 1979.

[3] Ahlfors, L. V., and C. J. Earle: Lectures on quasiconformal mappings. - Wadsworth \& Brooks/Cole Advanced Books \& Software, Monterey, Calif., 1966.

[4] Beurling, A.: Mittag-Leffler lectures on complex analysis (1977-1978). - In: The collected works of Arne Beurling, edited by L. Carleson, P. Malliavin, J. Neuberger and J. Wermer, Birkhäuser, 1989.

[5] Bishop, C.: An explicit constant for Sullivan's convex hull theorem. - Preprint, 1999.

[6] Bishop, C. J.: A fast approximation to the Riemann map. - Preprint, 2003.

[7] Bishop, C. J.: Conformal mapping in linear time. - Preprint, 2007.

[8] Buum, H.: Biological shape and visual science. - J. Theoret. Biol. 38, 1973, 205-287.

[9] Bridgeman, M.: Bounds on the average bending of the convex hull boundary of a Kleinian group. - Michigan Math. J. 51, 2003, 363-378.

[10] Bridgeman, M., and R. D. CANary: From the boundary of the convex core to the conformal boundary. - Geom. Dedicata, 96:1, 2003, 211-240.

[11] Canary, R. D.: The conformal boundary and the boundary of the convex core. - Duke Math. J. 106:1, 2001, 193-207. 
[12] Canary, R. D., A. Marden, D. B. A. Epstein, W. P. Thurston, and S. J. Patterson: Fundamentals of hyperbolic geometry : selected expositions. - London Math. Soc. Lecture Note Ser. 328, Cambridge Univ. Press, 2006.

[13] Choi, H. I., S. W. Choi, and H. P. Moon: Mathematical theory of medial axis transform. Pacific J. Math. 181:1, 1997, 57-88.

[14] Duren, P. L.: Univalent functions. - Grundlehren Math. Wiss. 259, Springer-Verlag, New York, 1983.

[15] Epstein, D. B. A., and A. Marden: Convex hulls in hyperbolic spaces, a theorem of Sullivan, and measured pleated surfaces. - In: Analytical and geometrical aspects of hyperbolic spaces, University Press, 1987, 113-253.

[16] Epstein, D. B. A., A. Marden, and V. Markovic: Complex angle scaling. - In: Kleinian groups and hyperbolic 3-manifolds, London Math. Soc. Lecture Note Ser., Cambridge Univ. Press, 2003, 343-362.

[17] Epstein, D. B. A., A. Marden, and V. Markovic: Quasiconformal homeomorphisms and the convex hull boundary. - Ann. of Math. (2), 159:1, 2004, 305-336.

[18] FeiszLI, M.: Differential geometry of smooth hyperbolic convex hull boundaries. - Preprint.

[19] Gardiner, F.P., and N. LAkic: Quasiconformal Teichmüller theory. - Math. Surveys Monogr. 76, Amer. Math. Soc., Providence, R.I., 2000.

[20] Garnett, J. B., and D. E. Marshall: Harmonic measure. - New Math. Monogr., Cambridge Univ. Press, 2005.

[21] Hubbard, J. H.: Teichmüller theory and applications to geometry, topology, and dynamics. Matrix Editions, Ithaca, NY, 2006.

[22] Lehto, O.: Univalent functions and Teichmüller spaces. - Grad. Texts in Math. 109, SpringerVerlag, New York, 1987.

[23] LeOnARD, K.: Measuring shape space: epsilon-entropy, adaptive coding and two-dimensional shape. - PhD Thesis, Brown University, 2004.

[24] Pommerenke, Ch.: Boundary behaviour of conformal maps. - Grundlehren Math. Wiss. 299, Springer-Verlag, Berlin, 1992.

[25] Sharon, E., and D. Mumford: 2D-Shape analysis using conformal mapping. - Internat. J. Comput. Vision 70:1, 2006, 55-75.

[26] Stephenson, K.: Introduction to circle packing. - Cambridge Univ. Press, 2005.

[27] Sullivan, D.: Travaux de Thurston sur les groupes quasi-fuchsiens et les varietes hyperboliques de dimension 3 fibrees sur $S^{1}$. - In: Bourbaki Seminar 1979/80, Springer, Berlin, 1981, 196-214.

[28] Thurston, W. P., and S. Levy: Three-dimensional geometry and topology. - Princeton Math. Ser. 35, Princeton Univ. Press, Princeton, N.J., 1997. 